\title{
A TRIBUTE TO HERMANN KÜMMEL ON HIS 80TH BIRTHDAY
}

\author{
RAYMOND F. BISHOP \\ Dept. of Physics, UMIST, Manchester M60 1QD, UK \\ R.F.Bishop@umist.ac.uk
}

Received 13 July 2001

One of the undoubted highlights of the 11th International Conference on Recent Progress in Many-Body Theories was the special session on Wednesday, 11 July, 2001 dedicated to the coupled cluster method (CCM), in honour of the upcoming 80th birthday of its founder and staunchest advocate, Hermann Kümmel. It was a special pleasure on this occasion that both Hermann Kümmel and Fritz Coester, the co-founder of the CCM, could be present and give invited talks. It was felt to be especially appropriate to mark Hermann Kümmel's 80th birthday at one of the conferences in this series due to his strong involvement in the series since its inception. Indeed, the esteem in which he is held by the community has already clearly been indicated by his election some years ago to the office of Honorary President of the International Advisory Committee of the conference series.

Hermann Kümmel was born in Berlin on 7 October, 1922. He spent his childhood and adolescence in that city, and graduated from high school (or 'Gymnasium') there in 1941 with his graduation diploma (or 'Abitur'). Upon graduating he was immediately drafted into the army, and his period of military service ended in 1945 . He then spent a year as a prisoner of war before he was able to start his university education. I will touch briefly again on this period toward the end of this tribute, but for the moment let us continue with the unfolding of his career.

In 1946 Hermann Kümmel began the study of physics at Humboldt University in East Berlin. His studies there in both theoretical and experimental physics led to the award of the Diploma degree in 1950, including an experimental study on the conductivity of organic fluids. In 1950 he began his higher graduate studies, now moving to the Free University in West Berlin, to work under Professor Ludwig. Here, for the first time, he meets the subject that will dominate his research thereafter, namely, quantum field theory. Two years later, in 1952, he obtained his $\mathrm{PhD}$ in Theoretical Physics, with a thesis dissertation which included the treatment of several problems in quantum electrodynamics. 
From 1952-57 Hermann Kümmel continued to work with Ludwig as an Assistant at the Free University in West Berlin. Papers published during this period cover subjects as diverse as

- the quantum theory of Boltzmann's equation,

- the connections between classical and quantum physics, and

- the Brueckner theory of atomic nuclei.

Toward the end of this period, in 1956, he received his Habilitation degree.

We now come to the year 1957-58 which Hermann Kümmel spent as a Research Associate at Iowa State University in Iowa City, USA. This is a key year both for him and the subject of the coupled cluster method, since it is during this period that, in collaboration with Fritz Coester, also then at Iowa State University, the foundations are laid for what they then called the "exponential- $S$ " approach to quantum field theory. These strong foundations will later solidify and develop into what is now known as the coupled cluster method (CCM) approach to microscopic quantum many-body theory, about which much more will be said later.

After a short stint as Dozent at the University of Tübingen after his return from the USA in 1958, Hermann Kümmel took up the joint positions of Assistant at the Max Planck Institute for Nuclear Chemistry in Mainz and Privatdozent at the University of Mainz. During the next few years some first applications of the CCM were made to

- the imperfect Fermi gas, and

- pairing effects in atomic nuclei.

After several years in Mainz, Hermann Kümmel returned to the USA in the early-to-mid 1960's to spend two years as a Professor of Physics at Oklahoma State University in Stillwater. He continued his work there on nuclear physics, including the study of direct nuclear reactions and pairing phenomena. At the end of this second trans-Atlantic interlude Hermann Kümmel returned to Mainz, now as Scientific Member (or senior scientist) at the Max Planck Institute and Adjunct Professor at the University of Mainz. From this point on he started to attract a group of students to him by teaching courses in theoretical physics at the university. He also started to build a strong research group at the Max Planck Institute. His main research at this time remained in nuclear physics, including

- the theory of nuclear decay,

- nuclear mass formulae,

- the theory of three-body systems, and

- parity-forbidden nuclear reactions.

1969 was a momentous year for Professor Kümmel. Tragically, his first wife, Mechthild (née Panick), died after 15 years of marriage which produced a son, Otto, born in 1955. He also moved, for the last time academically, with his entire research group to a Chair in Physics ('Lehrstuhl II') at the then brand-new Ruhr 
University in Bochum (RUB). The following year, in 1970, he remarried, to Heide (née Krüner), his present wife. Over the ensuing few years both a daughter, Eva, and a son, Tilmann, were born.

At the beginning of his stay in Bochum, much time was spent on getting the new university, RUB, established. Over the following years, however, Hermann Kümmel established RUB as one of the leading centres worldwide in quantum many-body theory, especially nuclear and subnuclear microscopic many-body theory studied via the coupled cluster method (CCM). Particularly, noteworthy are the very early attempts to use the CCM as a high-precision tool to obtain very accurate correlated nuclear wave functions to study parity impurities in nuclei. Very interestingly, this is a theme which will find resonance much later in the quantum chemistry community in their attempts to use the CCM and other methods to obtain high-precision atomic wave functions to study parity violation in atoms.

Apart from some sabbatical periods of a year or so in both the USA (Argonne and Stony Brook) and Taiwan, Professor Kümmel remained full-time at RUB until January 1988 when he retired at the mandatory retirement age of 65 . Thereafter, he has retained his association with RUB as Professor Emeritus.

From around 1965 there have been many excellent students and postdoctoral researchers working with Professor Kümmel. An incomplete list would include the following:

- Martin Altenbokum

- Kurt Emrich

- Wolfgang Ey

- Michael Fink

- Martin Funke

- Manfred Gari

- H. Hahn

- G. Hasberg

- Hans Hebach

- Ulrich Kaulfuss

- Karl-Heinz Lührmann

- Rainer Offermann

- Bernd Sommer

- Ude Wambach

- John Zabolitzky

Especially important during the earlier part of this Bochum period were the careful, accurate, and converged calculations on atomic nuclei using the CCM, work on which was restarted around 1971-72. Both ground- and excited-state properties were investigated of both closed-shell nuclei and open-shell nuclei containing one or two valence nucleons or valence holes. These calculations were, in many ways, all at least 10-15 years ahead of their time. Indeed, even at the present time, considerably 
less ambitious, inferior, and manifestly unconverged nuclear structure calculations are still being performed by others who have not yet appreciated what the Bochum group did a quarter of a century or so ago using the CCM!

These nuclear physics results of the 1970's in Bochum were, with hindsight, incredibly deep and powerful. Two of Hermann Kümmel's young colleagues, namely, Karl-Heinz Lührmann and John Zabolitzky, also deserve special mention for their important contributions to this project. Lührmann particularly distinguished himself by his deep insight into developing the theoretical basis of the CCM as a practical tool, while Zabolitzky was indispensable for so ingeniously solving the resulting nonlinear equations at a time when computers were unutterably limited in power by modern standards. With hindsight, John Zabolitzky was undoubtedly one of the best computational physicists in the world at a time when that term didn't even yet exist. There was probably nobody else in the world then who had the skill and resources available to solve the CCM equations involved in this research. Indeed, it is still a wonder to many of us in the quantum many-body theory and theoretical nuclear structure physics communities that around 25 years ago calculations were being done which included all of two- and three-body correlations properly done (i.e., all of renormalized Brueckner-Hartree-Fock, BruecknerBethe-Goldstone, and Bethe-Faddeev theory, plus more), plus the most important Bethe-Faddeev-Yakubovsky four-body terms - all of this at a time when computing power was a minute fraction of what it is today. Such calculations were clearly only attainable by both exploiting the available machines to the limits of their capability, and by extremely careful and clever coding.

The period 1969-88 of Kümmel's full-time employment at RUB, which coincides largely with the period of his development and application of the CCM, breaks reasonably accurately into two distinct periods, namely

- the 1970's: the nuclear era, and

- the 1980's: the subnuclear era.

A flavour of the work in the 1970's nuclear era is perhaps most economically conveyed by listing below the titles (and year of publication) of a representative sample of the published papers from this period. (Full publication details can be found in the appended publication list of Hermann Kümmel: the superscript numbers after the titles below indicate the numbers of the articles in that list.)

- Theory of many-body wave functions with correlations (1971), ${ }^{40}$

- Equations for linked clusters and the energy variational principle (1972), ${ }^{50}$

- Fully self-consistent Brueckner-Hartree-Fock and renormalized BruecknerHartree-Fock calculation for ${ }^{4} \mathrm{He}$ and ${ }^{16} \mathrm{O}(1973),{ }^{51}$

- Rearrangement energies in ground-state calculations (1973), ${ }^{52}$

- Equations for linked clusters and Brueckner-Bethe theory (1972), ${ }^{53}$

- Is the nucleus a many-body problem? (1974), ${ }^{57}$

- Degenerate many-fermion theory in $\exp (S)$ form $(1976),{ }^{60}$ 
- Comparison of the structure of Jastrow and exact many-fermion theories (1976), ${ }^{61}$

- Many-fermion theory in $\exp (S)$ (or coupled cluster) form (1978), ${ }^{62}$

- Maximum overlap and shape instabilities (1979), ${ }^{63}$ and

- Coupled cluster many-body theory in a correlated basis (1980). ${ }^{65}$

Most of the above papers are characterized by depth, insight, authority, and brevity. They repay repeated re-reading and merit close textual analysis. Some of the qualities and depth of this work can be surmised from the following quotation from the Introduction of the third of the above papers: ${ }^{51}$

"In this paper the $e^{S}$ version of the many-body theory will be used in the exact two-body correlation approximation and applied in the calculation of the binding energy (BE) and rms radius $\left\langle r^{2}\right\rangle$ of ${ }^{4} \mathrm{He}$ and ${ }^{16} \mathrm{O}$. This is done for several NN potentials (always including the Coulomb interaction), with and without the small terms mentioned before, c.m. corrections (including the exact one) and renormalization. From this, final conclusions can be drawn whether or not the potentials and/or the restriction to two-body correlations suffice to explain data, since everything else is exact."

In many ways this 1970's era of nuclear physics work by the Kümmel group culminated with the 1978 Physics Reports paper ${ }^{62}$ that draws all of the strands of the nuclear CCM work together. It is a masterful paper, but one which is an uncommonly challenging read. It is the sort of paper that one can only begin to appreciate properly years after the work has appeared, and after the same ground has been gone over many times by others.

After this decade of incredibly successful applications of the CCM to nuclear structure, Kümmel turned his attention in the 1980's to applying the method to subnuclear physics and quantum field theory. A flavour of the work in this subnuclear era can again be conveyed by a corresponding list to the one above of titles of a typical sample of published papers from this period:

- Coupled cluster description of relativistic many-body systems (1981), ${ }^{66}$ $\{R P M B T-2\}$,

- Effective operators in the relativistic meson-nucleon system (1983), ${ }^{67}$

- Expectation values and density matrices in the coupled-cluster theory (1983), ${ }^{68}$

- Coupled cluster description of field theories: procedures and their application to the vacuum sector in $(1+1)$-dimensional $\Phi^{4}$ field theories $(1985),{ }^{70}$

- Soliton sector of the $\left(\Phi^{4}\right)_{2}$ quantum field theory in the Hartree approximation (1985), ${ }^{71}$

- Coupled cluster approach to field theories (1985), ${ }^{73}\{R P M B T-4\}$,

- Coupled cluster description of pion-nucleon systems (1986), ${ }^{74}$

- A temperature-dependent coupled cluster method (1986), ${ }^{75}$

- Approaching the critical region of two-dimensional $\Phi^{4}$ field theory with postGaussian approximations (1987), ${ }^{77}$ 
- Convergence and critical phenomena studies in $\Phi_{1+1}^{4}$ field theories via the coupled cluster method (1987), ${ }^{80}\{R P M B T-5\}$,

- Using the coupled-cluster method in relativistic quantum field theories: a review (1988), ${ }^{81}$ and

- Summary of the 6th International Conference on Recent Progress in Many-Body Theories (1989), ${ }^{82}\{R P M B T-6\}$.

As indicated, four of the above papers from this subnuclear era have appeared in volumes of the proceedings of previous conferences in the present series of International Conferences of Recent Progress in Many-Body Theories.

Once again, one can convey some of the flavour of this work from the subnuclear era by focussing on a typical paper, ${ }^{74}$ whose Abstract reads (in full) as:

"The (nonperturbative) coupled cluster method is applied to the pion-nucleon system. After solving the vacuum and one-nucleon problem, a reasonable description of the deuteron is obtained."

The Hamiltonian density of the system is taken as the usual isospin-invariant pseudoscalar coupling model,

$$
\begin{gathered}
\mathcal{H}=\mathcal{H}_{0}+\mathcal{H}_{\text {int }}, \\
\mathcal{H}_{0}=\mathcal{H}_{0}(\mathbf{x})=\frac{1}{2}\left[\Pi_{t}^{\dagger} \Pi_{t}+\nabla \Phi_{t}^{\dagger} \cdot \nabla \Phi_{t}+m^{2} \Phi_{t}^{\dagger} \Phi_{t}\right]+\bar{\Psi}_{t^{\prime}}(-i \gamma \cdot \nabla+M) \Psi_{t^{\prime}}, \\
\mathcal{H}_{\text {int }}=\mathcal{H}_{\text {int }}(\mathbf{x})=-i g \int d^{3} x^{\prime} F\left(\mathbf{x}-\mathbf{x}^{\prime}\right) \bar{\Psi}_{t^{\prime}}(\mathbf{x}) \gamma_{5} \tau_{t} \Psi_{t^{\prime}}(\mathbf{x}) \Phi_{t}\left(\mathbf{x}^{\prime}\right),
\end{gathered}
$$

where $\Phi_{t}=\Phi_{t}(\mathbf{x})$ and $\Pi_{t}=\Pi_{t}(\mathbf{x})$ are respectively the pion (bosonic) field operator and its conjugate momentum density operator, and where $\Psi_{t^{\prime}}(\mathbf{x})$ and $\bar{\Psi}_{t^{\prime}}(\mathbf{x})$ are the four-component Dirac nucleon (fermionic) field operator and its adjoint respectively. The matrices $\gamma$ and $\gamma_{5}$ are the usual $(4 \times 4)$ Dirac matrices; the three matrices $\tau_{t}$ are the usual $(2 \times 2)$ Pauli isospin matrices; and the summation convention is implied over the repeated isospin indices $t^{\prime}$ for the (isospin- $\frac{1}{2}$ ) nucleon and $t$ for the (isospin1) pion. The form factor $F(\mathbf{x})$ is necessary to renormalize the pion-nucleon vertex. It is taken to have the standard form whose Fourier transform in momentum space is given as

$$
F(\mathbf{q})=\frac{\lambda^{2}-m^{2}}{\lambda^{2}+q^{2}},
$$

where $\lambda$ is a high-momentum (or small-distance) cutoff parameter. Finally, the masses $m$ and $M$ are the bare pion and nucleon masses in the theory.

Hasberg and Kümmel performed a CCM calculation for this system, ${ }^{74}$ in the multireference ("open-shell") formulation of the method. In the first place, the physical vacuum $|\Psi\rangle$ is written in terms of the bare vacuum $|\Phi\rangle$ in the usual CCM exponentiated form,

$$
|\Psi\rangle=e^{S}|\Phi\rangle,
$$


in terms of a correlation operator $S$ which is now expanded as a double sum,

$$
S=\sum_{m, n=0}^{\infty} S_{m n}, \quad m+n \geq 1,
$$

in terms of the number $m$ of virtual pions and the number $n$ of virtual nucleonantinucleon pairs. Secondly, the (physical) one-nucleon state is treated by analogy to the one-valence open-shell system,

$$
|N(\mathbf{p})\rangle=e^{S}\left(1+F^{(1)}\right) a^{\dagger}(\mathbf{p})|\Phi\rangle,
$$

where $a^{\dagger}(\mathbf{p})$ creates a nucleon with momentum p. Thirdly, and finally, the twonucleon state is treated by analogy to the two-valence open-shell system,

$$
\begin{aligned}
\left|N_{1}, N_{2} ; \alpha\right\rangle= & \int d^{3} p_{1} \int d^{3} p_{2}\left[e^{S}\left(1+F^{(1)}+\frac{1}{2}: F^{(1)^{2}}:+F^{(2)}\right)\right] \\
& \times a^{\dagger}\left(\mathbf{p}_{1}\right) a^{\dagger}\left(\mathbf{p}_{2}\right)|\Phi\rangle C_{\alpha}\left(\mathbf{p}_{1}, \mathbf{p}_{2}\right),
\end{aligned}
$$

where the factor $\frac{1}{2}$ in the quadratic term describing two "dressed ", or physical, but uncorrelated nucleons prevents us from counting each twice; and this term is also normal-ordered so as to avoid contractions (or links) between them, which are more properly contained in the genuine two-nucleon correlation operator $F^{(2)}$. Both of the operators $F^{(1)}$ and $F^{(2)}$ are decomposed in a similar fashion to $S$ above.

Hasberg and Kümmel ${ }^{74}$ performed such a multireference CCM calculation in which they kept the partitions $S_{01}, S_{11}, F_{10}^{(1)}$ (and $F_{20}^{(1)}, F_{01}^{(1)}$, and $F_{11}^{(1)}$ in low order). For the two-nucleon problem only those terms containing amplitudes from the vacuum and the one-particle problem were included. This naturally includes the (most important) one-boson exchange terms.

Clearly, the results depend on the parameters $M, g$, and $\lambda$. In principle they also depend on the bare pion mass $m$, but the pion self-energy is actually a higher-order effect than the above approximations used, and hence $m$ is set equal to the physical (experimental) pion mass. The one-nucleon calculation was then used to fit the bare nucleon mass $M$ from the CCM prediction for the physical (experimental) nucleon mass. Hence, the two remaining parameters $\lambda$ and $g$ could be used to examine the two-nucleon (deuteron) binding energy. The dependence on $\lambda$ was, very satisfyingly, found to be weak, and with values of the pion-nucleon coupling constant $g^{2}$ close to the physical ("experimental") value, very good agreement could be found with the experimental deuteron binding energy. This is a remarkable result, especially since the convergence obtained in these pioneering calculations was impressive. As the authors themselves noted:

"One may ask how reliable these numbers are. All indications are that, indeed, the terms not included can be neglected: already in the approximation scheme we have used relatively few terms and all terms of higher order turned out to be numerically small. This is in line with the numerical experience in many-body theory. Roughly speaking it is due to the fact that chains (ladders) of infinite 
length have been summed. Only the number of particles excited "at the same time" is very much restricted."

Once again, this field-theoretic application of the CCM and others performed by Kümmel in his 1980's subnuclear era were many years ahead of their time. Even now, very few more ambitious (and manifestly converged) calculations have ever been undertaken for such strongly interacting systems. Although he retired in 1988, when he was succeeded in his Chair at RUB by Klaus Goeke, Professor Kümmel has continued to work as an Emeritus Professor on applications of the CCM, especially to quantum field theory. Indeed, his latest paper ${ }^{87}$ appeared in the month of this conference. One sees, therefore, that Hermann Kümmel's work spans the full half-century since his first publication in 1950.

Since the CCM and its applications are discussed in detail by Kümmel himself and by other contributors to this volume, I will content myself here with just two important comments concerning the CCM which Kümmel pioneered, and which is both so closely identified with him and so inextricably woven into his long career. Firstly, one should recall the enormous range of successful applications of the CCM that have been made since its conception in 1957-58, both in the form in which it was invented, the so-called normal coupled cluster method (NCCM), and in its later extended (ECCM) version. Without any attempt to be exhaustive, we note that some of the most important applications of the CCM have been to the following systems:

- Atomic Nuclei: The NCCM has very successfully been applied to the ground and excited states of both closed-shell nuclei (e.g., ${ }^{4} \mathrm{He},{ }^{16} \mathrm{O},{ }^{40} \mathrm{Ca}$ ) and the open-shell nuclei (e.g., ${ }^{15} \mathrm{~N},{ }^{17} \mathrm{O},{ }^{14} \mathrm{C},{ }^{18} \mathrm{O},{ }^{18} \mathrm{~F}$ ) formed by the addition to the closed-shell nuclei of one or two valence particles or holes. Calculations have been performed for a variety of phenomenological two-body (and three-body) internucleon potentials, in a hard-core truncation scheme that includes up to 4-body correlations. Calculated quantities include ground-state energies, density distributions, elastic-scattering electron form factors, and excitation spectra. Numerical convergence has been explicitly demonstrated at the above four-body correlation level, and no other ab initio technique has ever bettered (or even equalled) these results.

- Nuclear Matter: Very similar calculations have also been performed, starting with the work of Day and his collaborators, for nuclear matter, with similarly converged results for the binding energy per nucleon and the saturation density, for a range of phenomenological internucleonic forces.

- Atoms and Molecules: The CCM has become the method of choice in quantum chemistry wherever high accuracy is required. A huge range of calculations has been performed on such quantities as ionization potentials, electron affinities, Auger spectroscopy, excitation energies, and energy gradients (for use, for example, in searching potential energy surfaces to predict vibrational spectra or to locate transition states in decomposition reactions). Very many atoms and molecules (including, e.g., $\mathrm{LiH}, \mathrm{H}_{2} \mathrm{O}$, GaAs, benzene, s-tetrazine, and many, 
many others) have been studied, and state-of-the-art calculations are now done on molecules with up to 100 or more active electrons. Especially for the heavier atoms, calculations have been performed with relativistic and QED corrections incorporated.

- The Electron Gas: The NCCM has been applied to the electron gas in the high-density (random phase approximation) limit, in the intermediate metallic density range, and in the low-density (Wigner crystal) regime. Results in the important metallic density range for the correlation energy, for example, are accurate to better than 1 milli-Hartree per electron (or $<1 \%$ ) by comparison to the essentially exact Green's function Monte Carlo results available. This accuracy has never been equalled in any other $a b$ initio calculation of what is still one of the best studied of all quantum many-body systems.

- Charged Impurity in a Polarizable Medium: The technique of allowing low-energy positrons to annihilate inside metals, alloys, and other forms of condensed matter has become an important experimental tool to probe these materials. Such systems comprising a charged impurity in a polarizable medium are prototypes of field-theoretical one-body problems. By casting the polarization degrees of freedom as internal gauge fields, it has been shown how the ECCM can provide a powerful microscopic description of these systems.

- Quantum Fluid Mechanics: By coupling to external gauge fields (viz., the $\mathrm{U}(1)$ scalar and vector potentials), which serve to set the system into motion, it has been shown how the ECCM can provide a very complete, microscopic description of the zero-temperature quantum hydrodynamics of a stronglyinteracting condensed Bose fluid. Inter alia, a hierarchy of exact local balance equations (for number conservation, momentum conservation, energy conservation, etc.) has been derived microscopically. These are precisely the quantummechanical macroscopic laws of hydrodynamics for the system. Furthermore, the well-known Gross-Pitaevskii approximation for this system is regained at the lowest $(\mathrm{SUB}(1))$ level of implementation of the CCM.

- Quantum Spin Lattice Models of Magnetism: Extensive and very successful applications of the CCM have been made in recent years to a wide variety of quantum spin lattice models. Examples include the solid phases of ${ }^{3} \mathrm{He}$; the isotropic (Heisenberg) and anisotropic $X X Z$ models in one dimension and on various two-dimensional lattices (including the square, triangular and hexagonal lattices), both for spin-half and higher-spin systems; the spin-one Heisenbergbiquadratic model; and to such frustrated spin models as the spin-half $J_{1}-J_{2}$ model; and the two-dimensional (anisotropic) Heisenberg antiferromagnet on a triangular lattice. The CCM has also been applied to the spin-one easy-plane ferromagnet; to a model which interpolates between the Heisenberg models on the triangular and kagomé lattices; and to many other equally or more complicated examples.

- Electron Lattice Models: The CCM has been applied to such lattice models of strongly interacting electrons as the Hubbard model, both precisely at halffilling and with the further removal of one or two electrons. 
- Anharmonic Oscillators: Both the NCCM and ECCM have been intensively applied to anharmonic oscillator systems considered as $(0+1)$-dimensional models of nonlinear quantum field theories. The structures of both the NCCM and ECCM have been particularly explored by applications to such models of the powerful holomorphic (or Bargmann) representation. Analogous anharmonic spin models have also been studied as corresponding models in finite-dimensional Hilbert spaces.

- Continuum Quantum Field Theories: Kümmel and his co-workers have very successfully applied the NCCM to several continuum quantum field theories. These include: (i) $\Phi^{4}$ field theory in $1+1$ and $2+1$ dimensions, in the vacuum (ground state) and one-particle (mass gap) sectors, and in the soliton sector; (ii) $\Phi^{4}+\Phi^{6}$ field theory in $1+1$ dimensions, in the vacuum, one- and two-particle sectors; (iii) the sine-Gordon model in $1+1$ dimensions in the vacuum, oneparticle and soliton sectors; and (iv) the vacuum, one- and two- particle sectors of the model $(3+1)$-dimensional field theory of interacting pions and nucleons discussed above. Some preliminary applications of the ECCM to quantum field theory have also been made, using the sine-Gordon model as an illustration.

- Lattice Quantum Field Theories: The NCCM has been applied to various lattice gauge field theories. These include the discrete $\mathrm{Z}(2)$ model, and such continuous groups as the Abelian U(1) model of lattice QED and the nonAbelian $\mathrm{SU}(2)$ case. Attempts have also been made to study $\mathrm{SU}(n)$ lattice field theories with $n \geq 3$. Both the NCCM and ECCM have also been successfully applied to a latticised $\mathrm{O}(4)$ nonlinear sigma model of chiral meson field theory, especially to study the chiral phase transition.

- Quantum Optics: Recent applications of the NCCM have been made to some prototypical models of interacting fermions (or spins) and bosons which occur in quantum optics and related fields. These include, for example, the Rabi Hamiltonian (or Jaynes-Cummings model without the rotating wave approximation).

- Solid-State Optoelectronics: Similar applications of the NCCM have been made to the linear $E \otimes e$ Jahn-Teller effect, and to related model non-adiabatic Hamiltonians.

- Other Model Many-Body Problems: Various other applications of the CCM have been made to a variety of important model problems. Examples include: (i) the Lipkin-Meshkov-Glick SU(2) quasispin model of the spherical to deformed shape transition in the rare-earth nuclei under rotation; (ii) the exactly integrable one-dimensional Lieb model of bosons interacting via pairwise repulsive Dirac delta-function potentials; and (iii) the polaron problem.

Secondly, and most importantly in such a historical survey of the CCM, one would be remiss not to mention also the role of Fritz Coester in the conception of the method. There is no doubt whatsoever that he first planted the seed which became the CCM, and which then flourished so well in the hands of Kümmel and his collaborators. There is equally no doubt that it would have been entirely appropriate 
to pay tribute to both Coester and Kümmel on the occasion of their 80th birthdays at the special session devoted to the CCM. However, those who know Fritz Coester will not be surprised to learn that, although invited by the International Advisory Committee to share the honours with Hermann Kümmel on this occasion, he declined to do so on the grounds that it would detract from what in his view was the much more important role of Kümmel in developing and applying the method. Although we all admired and respected his decision, it was nonetheless a source of great pleasure to all present that he contributed to the session and to this volume.

Before concluding it would perhaps be appropriate to devote some words to Hermann Kümmel's human qualities. As all who know him well will attest, he is a man of great charm, erudition and modesty. He is also a man of high moral principles. Both of his parents were historians of Far Eastern art, and much of their interest has rubbed off on him. Like Fritz Coester he has never enjoyed blowing his own trumpet, and he has never sought undue advancement or self-aggrandisement. Indeed, on occasions, in that subtle way the world has of surprising us, this has even worked greatly to his advantage. For example, he was the only one in his high-school class of 1941 who, when drafted into the army, did not make at least the rank of junior officer. This was good luck indeed, since most such young officers were killed in action. During World War 2 he also spent a few weeks in military prison, because a letter to his brother complaining about the government was opened. His fate for that could also have been far worse. Indeed, as Hermann himself has observed, it is only due to such a series of rare lucky incidents that he survived at all. On the other hand, perhaps great men, like great generals, also make their own luck.

In conclusion let us ask how this scientific career of some 50 years of Hermann Kümmel may be characterised. In the first place it is obvious that his entire scientific output has been devoted to basic research (or what we now often call "blue skies research") of the very highest order. He comes from a generation where quality is prized far beyond quantity. At this point it is also worth recalling the freedom that institutions like the Max Planck Institutes (MPI) offered outstanding scientists like Hermann Kümmel to prosecute their research totally unfettered by considerations of relevance or usefulness, or judged against criteria like benefiting the economic or social and industrial progress of the nation, and where the publish-or-perish atmosphere simply didn't exist. Indeed, Hermann has recalled on several occasions how this lack of any obligations placed on him, and the lack of any need to compete for funds, or to justify his research in any way while at the MPI in Mainz, gave him a truly golden opportunity to pursue his long-term scientific goals that have since borne so much fruit.

\section{References}

1. H. Kümmel, Der Energie-Impulsvektor in der unitären divergenzfreien quantenelektrodynamik, Z. Naturforsch. 5a, 642 (1950).

2. H. Kümmel, Freie elektronen in der unitären quantenelektrodynamik, Z. Phys. 134, 78 (1952). 
3. H. Kümmel, Zur feldtheoretischen beschreibung des festen körpers, Z. Naturforsch. 9a, 331 (1954).

4. H. Kümmel, Die wechselwirkung vieler teilchen, I. allgemeine theorie, Z. Naturforsch. 10a, 117 (1955).

5. H. Kümmel, Quantentheoretische begründung der Boltzmann'schen stoßgleichung, Z. Phys. 143, 219 (1955).

6. H. Kümmel, Zur quantentheoretischen begründung der klassischen physik, I. dynamik der gase und flüssigkeiten, Nuovo Cim., Ser. X 2, 1057 (1955).

7. H. Kümmel, Zur quantentheoretischen begründung der klassischen physik, II. statistische mechanik und thermodynamik, Nuovo Cim., Ser. X 2, 877 (1955).

8. H. Kümmel, Boltzmann-gleichung und thermodynamik, Z. Phys. 145, 496 (1956).

9. H. Kümmel, Irreversibilität und quantentheorie, Z. Naturforsch. 11a, 15 (1956).

10. H. Kümmel, Die eigenschaften der quantentheoretischen phasenraumdichte, Nuovo Cim., Ser. X 3, 870 (1956).

11. H. Kümmel, Zur begründung der Brueckner'schen theorie des atomkerns, Z. Naturforsch. 12a, 85 (1957).

12. H. Kümmel, Ein störungstheoretischer zugang zu der brueckner'schen theorie des atomkerns und der Watson'schen streunäherung, Nuovo Cim., Ser. X 6, 23 (1957).

13. F. Coester and H. Kümmel, Time dependent theory of scattering of nucleons by nuclei, Nucl. Phys. 7, 421 (1958).

14. F. Coester and H. Kümmel, Short-range correlations in nuclear wave functions, Nucl. Phys. 17, 447 (1960).

15. H. Kümmel, On the pairing energy of medium heavy nuclei, Z. Naturforsch. 16a, 208 (1961).

16. H. Kümmel, Compound pair states in imperfect Fermi gases, Nucl. Phys. 22, 177 (1961).

17. H. Kümmel, Grundzustand eines Fermi-gases mit hard-sphere wechselwirkung, Z. Phys. 166, 243 (1961).

18. H. Kümmel, Non perturbational linked cluster expansion of the Fermi gas wave function, in Lectures on Many Body Theory (Academic Press, New York, 1962), p. 265.

19. H. Kümmel, J. H. E. Mattauch, W. Thiele and A. H. Wapstra, A new nuclidic mass law, in Proc. 2nd Conf. Nuclidic Masses, ed. Johnson, Jr. (Springer-Verlag, Wien, 1964), 42.

20. H. Hebach and H. Kümmel, Neutron-proton interaction and nuclear binding energy, Z. Phys. 186, 452 (1965).

21. H. Kümmel, A note on the theory of decaying systems and the computation of decay rates, Z. Naturforsch. 21a, 79 (1966).

22. H. Kümmel with J. L. Adams, Deformation energies and collective motion of heavy nuclei, Nucl. Phys. 80, 145 (1966).

23. H. Kümmel, J. H. E. Mattauch, W. Thiele and A. H. Wapstra, A new mass law with shell and deformation corrections, Nucl. Phys. 81, 129 (1966).

24. H. Hebach, P. Henneberg and H. Kümmel, Three-body model for ${ }^{6} \mathrm{He}$, Phys. Lett. B24, 134 (1967).

25. H. Kümmel, Recent development in direct reaction theory, Lectures at the Liperi Summer School, Helsinki, 1966.

26. K. Hoersch and H. Kümmel, Alpha-cluster probability in nuclear matter, Z. Phys. 203, 91 (1967).

27. W. M. Ewers and H. Kümmel, Three-body decay of the $1.71 \mathrm{MeV}(2+)$ state of ${ }^{6} \mathrm{He}$, Phys. Lett. B26, 480 (1968).

28. R. G. Beil and H. Kümmel, Inelastic electron scattering from nuclear matter, Z. Phys. 212, 248 (1968). 
29. H. Kümmel, Calculations of nuclear masses from first principles, in Proceedings of the Third Intern. Conf. on Atomic Masses, p. 20 (Univ. Manitoba Press, Winnipeg, 1967).

30. M. Gari and H. Kümmel, Parity forbidden alpha decay of some $0^{16}$ levels, Phys. Rev. Lett. 23, 26 (1969).

31. O. Dumitrescu, H. Kümmel and A. Sandulescu, On three particle stripping reactions, Z. Phys. 220, 45 (1969).

32. J. G. Zabolitzky, M. Gari and H. Kümmel, Short range correlations in a Woods-Saxon Shell-Model, Nucl. Phys. A155, 526 (1970).

33. H. Kümmel, Die struktur des atomkerns, Bild der Wissenschaft 121 (1971).

34. H. Kümmel with H. Hebach and P. Henneberg, Three-cluster models of some nuclei, in Intern. Conf. on Clustering Phenomena in Nuclei, Bochum, 21-24 July 1969 (Int. Atom. Energy Agency, Vienna, 1970).

35. U. Wambach and M. Gari and H. Kümmel, Circular polarization of gamma rays in ${ }^{18} \mathrm{~F}-\mathrm{A}$ test of weak interaction theories, Phys. Lett. B33, 253 (1970).

36. H. Kümmel, Short range correlations, lectures given at the Int. Summer School on Nuclear Reactions, Predeal, Romania, 1970; Com. Nat. Rom. d. Fiz., 37 (1971).

37. M. Gari, H. Kümmel and J. G. Zabolitzky, Parity violating effects in nuclei, lectures given at the Intern. Summer School on Nuclear Reactions, Predeal, Romania, 1970; Com. Nat. Rom. d. Fiz., 38 (1971).

38. M. Fink, H. Hebach and H. Kümmel, Note on short range correlations and elastic electron scattering, Phys. Rev. C3, 2075 (1971).

39. M. Gari, H. Kümmel and J. G. Zabolitzky, Short range correlations and parity impurities, Nucl. Phys. A161, 625 (1971).

40. H. Kümmel, Theory of many body wave functions with correlations, Nucl. Phys. A176, 205 (1971).

41. H. Kümmel, Atom nuclei parity and time reversal invariance, Verh. Deutsch. Phys. Ges. 11, 842 (1971).

42. H. Kümmel with M. Fink, M. Gari, H. Hebach, J. Schlitter and J. G. Zabolitzky, Berechnung kurzreichweitiger korrelationen in atomkernen, in Fachberichte Physikertagung, Hannover, 1970. (Teubner-Verlag, 1970).

43. M. Gari, O. Dumitrescu, J. G. Zabolitzky and H. Kümmel, A first step towards realistic calculation of parity-mixing effects in heavy nuclei, Phys. Lett. B35, 19 (1971).

44. O. Dumitrescu and H. Kümmel, Resonance scattering theory and the structure of compound states, Ann. Phys. (NY) 71, 556 (1971).

45. H. Kümmel, Calculation of parity-violating effects in nuclei, in International Symposium on Nucleons and Weak Interactions, Zagreb 1971, ed. E. Tadic (Zagreb, 1972).

46. M. Fink, H. Hebach and H. Kümmel, On elastic electron scattering and short range correlations, reply to the note by C. Ciofi degli Atti, Phys. Rev. C5, 1434 (1972).

47. M. Fink, H. Hebach and H. Kümmel, Calculations on $(\gamma, p)$ and $(\gamma, n)$ reactions at photon energies between 40 and $100 \mathrm{MeV}$, Nucl. Phys. A186, 353 (1972).

48. H. Kümmel, Electromagnetic test of high momentum components in nuclear wave functions, J. de Phys. I 33, 127 (1972).

49. M. Fink, H. Hebach, J. Schlitter and H. Kümmel, Elastic electron scattering with short range correlations in ${ }^{4} \mathrm{He}$ and ${ }^{16} \mathrm{O}$, Phys. Lett. B39, 147 (1972).

50. H. Kümmel and K. H. Lührmann, Equations for linked clusters and the energy variational principle, Nucl. Phys. A191, 525 (1972).

51. H. Kümmel and J. G. Zabolitzky, Fully self-consistent Brueckner-Hartree-Fock and renormalized Brueckner-Hartree-Fock calculation for ${ }^{4} \mathrm{He}$ and ${ }^{16} \mathrm{O}$, Phys. Rev. C7, 547 (1973). 
52. H. Kümmel, Rearrangement energies in ground state calculations, Phys. Rev. C8, 1144 (1973).

53. K. H. Lührmann and H. Kümmel, Equations for linked clusters and Brueckner-Bethe theory, Nucl. Phys. A194, 225 (1972).

54. O. Dumitrescu, M. Gari, H. Kümmel and J. G. Zabolitzky, Circular polarization of gamma rays and parity nonconserving interactions, Z. Naturforsch. 27, 733 (1972).

55. H. Kümmel, W. Schelongowski and J.G. Zabolitzky, BHF-theories - approach to reality? In International Symposium on Correlations in Nuclei, Balatonfüred, Hungary, 1973.

56. H. Kümmel, Erkenntnisforschung und anwendungsforschung, Bild der Wissenschaft 10, 1494 (1973).

57. H. Kümmel, Is the nucleus a many-body problem? In Symposium on Mesonic Effects in Nuclear Structure in Remembrance of K. Erkelenz, Bonn, 1974.

58. H. Kümmel, The nucleus a many body system — truth or wishful thinking? In Interaction Studies in Nuclei, eds. H. Joachim and B. Ziegler (North Holland, Amsterdam, 1975), p. 133.

59. H. Kümmel, K. H. Lührmann and J. G. Zabolitzky, The many body theory for finite nuclei (and other Fermi systems), in Int. Conf. on self consistent fields, Trieste (North Holland, Amsterdam, 1975), p. 195.

60. R. Offermann, H. Kümmel and W. Ey, Degenerate many fermion theory in $\exp (S)-$ form, Nucl. Phys. A273, 349 (1976).

61. H. Kümmel, Comparison of the structure of jastrow and exact many fermion theories, Z. Phys. A279, 271 (1976).

62. H. Kümmel, K. H. Lührmann and J. G. Zabolitzky, Many fermion theory in the $\exp S$-(or coupled cluster) form, Phys. Rep. 36C, 1 (1978).

63. H. Kümmel, Maximum overlap and shape instabilities, Nucl. Phys. A317, 199 (1979).

64. H. Kümmel, Nuclear masses from first principles, in 6th Int. Conf. On Atomic Masses, East Lansing, Michigan, 1979.

65. E. Krotscheck, H. Kümmel and J. G. Zabolitzky, Coupled cluster many body theory in a correlated basis, Phys. Rev. A22, 1243 (1980).

66. H. Kümmel, Coupled cluster description of relativistic many body systems, in Proceedings of th 2nd Int. Conf. On Recent Progress in Many Body Theories, Oaxtepec, Mexico, 1981, ed. J. G. Zabolitsky (Springer, Berlin 1981).

67. H. Kümmel, Effective operators in the relativistic meson-nucleon system, Phys. Rev. C27, 765 (1983).

68. H. Kümmel, Expectation values and density matrices in the coupled-cluster theory, Int. J. Quant. Chem. 24, 79 (1983).

69. H. Kümmel, The coupled cluster method, in Nucleon-Nucleon Interaction and Nuclear Many Body Problem Chanchun, China, 1983, eds. S. S. Wu and T. T. Kuo (World Scientific, Singapore, 1983).

70. C. S. Hsue, H. Kümmel and P. Ueberholz, Coupled cluster description of field theories: procedures and their application to the vacuum sector in $(1+1)$-dimensional $\Phi^{4}$ field theories, Phys. Rev. D32,1435 (1985).

71. M. Altenbokun and H. Kümmel, Soliton sector of the $\left(\phi^{4}\right)_{2}$ quantum field theory in the Hartree approximation, Phys. Rev. D32, 1435 (1985).

72. H. Kümmel, Preparing the ground for coupled cluster calculations, in 9th Int. Workshop on Condensed Matter Theories, San Francisco, 1985.

73. H. Kümmel, Coupled cluster approach to field theories, in 4th Int. Conf. On Recent Progress in Many Body Theories, San Fransisco, 1985.

74. G. Hasberg and H. Kümmel, Coupled cluster description of pion-nucleon systems, Phys. Rev. C33, 1367 (1986). 
75. M. Altenbokum, K. Emrich, H. Kümmel and J. G. Zabolitzky, A temperature dependent coupled cluster method, in Condensed Matter Theories, Vol. 2, eds. P. Vashishta, R. K. Kalia and R. F. Bishop (Plenum Press, New York, 1987), p. 389.

76. M. Altenbokum, K. Emrich, H. Kümmel and J. G. Zabolitzky, Treatment of the Bloch equation by the coupled cluster method, Nuovo Cim. B97, 41 (1987).

77. M. Funke, U. Kaulfuß and H. Kümmel, Approaching the critical region of twodimensional $\Phi^{4}$ field theory with post-gaussian approximations, Phys. Rev. D35, 621 (1987).

78. R. F. Bishop and H. Kümmel, The coupled-cluster method, Physics Today 40(3), 52 (1987).

79. H. Kümmel, The anharmonic oscillator revisited, in Condensed Matter Theories, Vol. 3, eds. J. S. Arponen, R. F. Bishop and M. Manninen (Plenum Press, New York, 1987), p. 21.

80. H. Kümmel, Convergence and critical phenomena studies in $\Phi_{1+1}^{4}$ field theories via the coupled cluster method, in Recent Progress in Many-Body Theories, Vol. 1, eds. A. J. Kallio, E. Pajanne and R. F. Bishop (Plenum Press, New York, 1988), p. 265.

81. H. Kümmel, Using the coupled-cluster method in relativistic quantum field theories, a review, in Workshop on Relativistic Nuclear Many Body Physics Columbia, 1988.

82. H. Kümmel, Summary of the 6 th international conference on recent progress in manybody theories, in Recent Progress in Many-Body Theories, Vol. 2, ed. Y. Avishai (Plenum Press, New York, 1990), p. 353.

83. H. Kümmel, Origins of the coupled cluster method, Theor. Chim. Acta 80, 81 (1991).

84. M. Funke and H. Kümmel, Low energy states of $(1+1)$-dimensiona1 $\Phi^{6}$ field theories via the coupled cluster method, Phys. Rev. D30, 991 (1994).

85. H. Kümmel, Higher order coupled cluster approximations to the vacuum. of the $\Phi^{4}$ field theory in $1+1$ dimensions, Phys. Rev. D50, 6556 (1994).

86. H. Kümmel, Variational gaussian approximation to the soliton of the 1+1-dimensional sine-Gordon model, Phys. Rev. B58, 2620 (1998).

87. H. Kümmel, Post-gaussian approximation to the soliton of the $1+1$-dimensional sine-Gordon model, Phys. Rev. B64, 014301 (2001). 